\title{
Preliminary Results from a Sub-Ångstrom TEM
}

\author{
A.Genç*, J. Ringnalda**, D.M. Maher*, H. Colijn ${ }^{*}$, H.L. Fraser* \\ * Department of Materials Science and Engineering, The Ohio State University, 2041 \\ College Road, Columbus, Ohio, 43210 \\ ** FEI Electron Optics, PO Box 80066, 5600 KA Eindhoven, The Netherlands
}

This paper discusses the installation process of a new sub-Ångstrom capability Transmission Electron Microscope (TEM) at the Ohio State University. As the requirements for research approach smaller and smaller scales, new instrumentation with higher resolution becomes a necessity. The performance of these new systems is very dependent on the site where they are located. It can be stated that these new microscopes are the most sensitive microphone, the most sensitive seismometer and also the most sensitive magnetometer. As such the systems can be improved and made more stable, but still the site affects the final performance to a significant degree. The system at OhioState has been installed in a specially designed room that has minimal environmental instabilities. The microscope is located on a low-vibration platform, which is mechanically isolated from the rest of the building. Fig. 1 is a cross-section view of the support structure for the microscope. The overall structure of this platform is aimed to lower both the vertical and horizontal vibrations especially in the low frequency range (0$10 \mathrm{~Hz}$ ). For fine analytical probe performance, electromagnetic fields give rise to instabilities in the electron beam. For this reason, the reinforcement in the concrete is fiberglass rods instead of metal, and the electrical supplies in the room were all checked for ground loops and current imbalances. The vertical and horizontal AC magnetic fields around the column are reduced to values of less then $0.2 \mathrm{mG}$ [1]. The stability of the image and the microscope is very dependent on the thermal stability in the room. Measurements on-site now indicate a short-term stability in room temperature of less than $0.2^{\circ} / \mathrm{min}$ and long-term of less than $1^{\mathrm{o}} /$ day.

In this special location, the system achieves routine sub-Ångstrom resolution in the parallel illumination / high-resolution transmission electron microscopy (HRTEM) mode and 1.36 Ångstrom resolution in the probe / scanning transmission electron microscopy (STEM) mode. Figure 2 a. is an image of the Young's fringes taken from the gold particles on a carbon film sample revealing the line resolution down to sub-Ångstrom level, $\sim 0.7 \AA$, Figure 2 b. is a Fast Fourier Transformation (FFT) taken from an Aluminum specimen showing the $0.064 \AA$ frequencies, c. is a high angular dark-field STEM image of the Si dumbbells at the $<110>$ orientation and $d$. is the drift test result addressing the thermal and pressure stabilities in the room environment.

\section{References}

[1] D.A. Muller et al., Journal of Electron microscopy, 50(3): 219-226 (2001) 


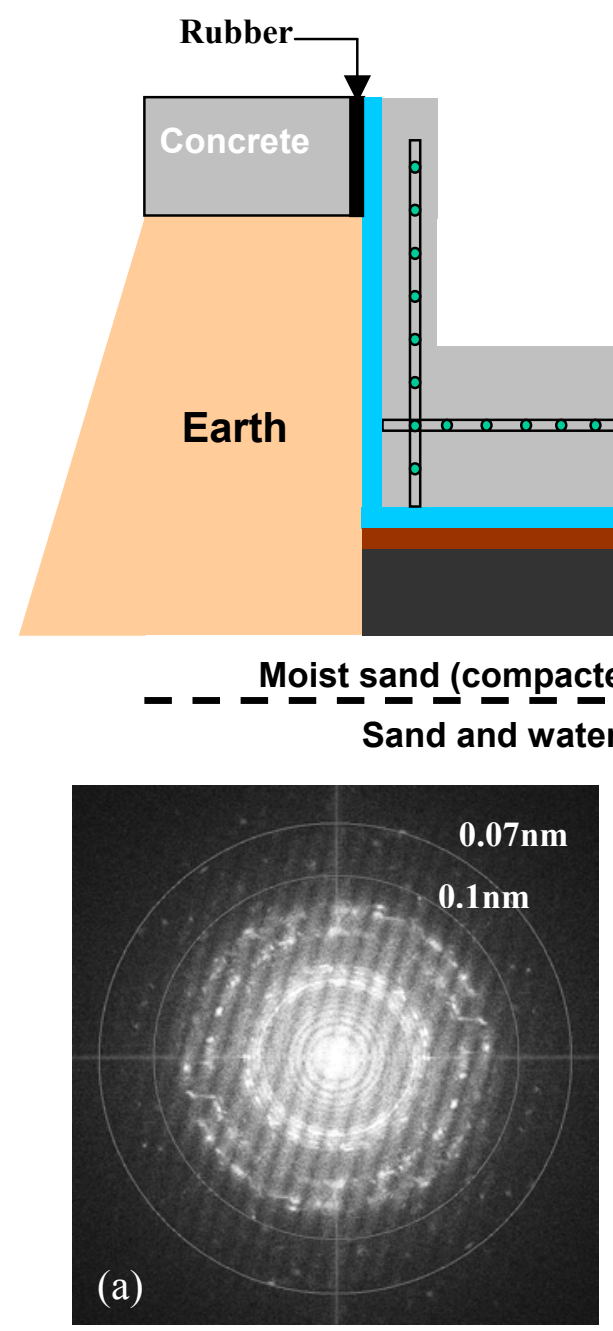

Fig.1 The cross-section view of the low-vibration platform designed for the $300 \mathrm{kV}$ TEM TITAN.
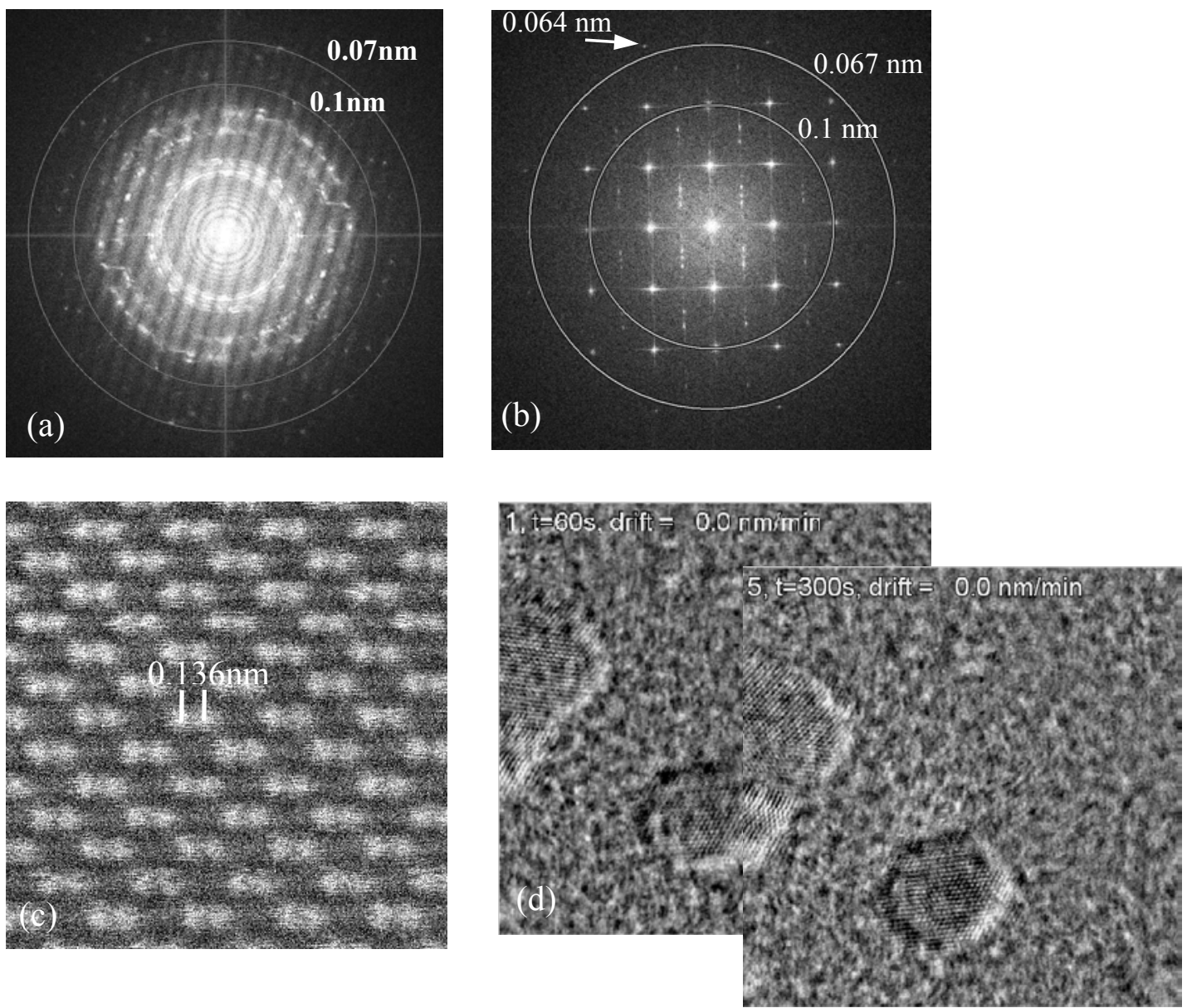

Fig.2 a. The Young`s fringes showing the sub-Ångstrom line resolution b. the FFT of an Al specimen, $0.064 \mathrm{~nm}$ frequencies are pointed out c. $<110>$ Si dumbbells are resolvable at the probe /STEM mode d. drift-test result, showing the amount of drift after $60 \mathrm{~s}$ as $0.0 \mathrm{~nm} / \mathrm{min}$ and after $300 \mathrm{~s}$ as $0.0 \mathrm{~nm} / \mathrm{min}$. 